\title{
Some Notes on the Method of Perfusing an Isolated Rabbit-heart
}

\author{
Sakuzi Kodama \\ The First Department of Physiology, \\ Kumamoto University School of Medicine, Kumamoto
}

\begin{abstract}
With the method of perfusing an isolated rabbit-heart recently reported by the author, the heart did not sufficiently manifest its activities in parallel recordings of pressures in the pulmonary artery and vein, aorta and vena cava, though the activities could be observed with the naked eye.

$\mathrm{B}_{y}$ slightly modifying the method and experimental procedures, the heart was found to manifest its activities sufficiently in the parallel recordings of the pressures during some three hours.
\end{abstract}

In a recent report, ${ }^{1}$ the author described a method of perfusing the isolated rabbit-heart, with a simultaneous pressure-recording of the aorta, vena cava, pulmonary artery and vein. In this method, blood-Ringer solution was used as the perfusate. The author could not obtain well-defined recordings of pressure in the four blood vessels, in spite of apparently sufficient activity of the heart. The author considered at first that these incomplete recordings would be due to some inadequacy of the perfusate which was possibly devoid of components essential for the cardiac activity. But in further experiments, the author found that the heart could manifest its activities sufficiently in pressure recordings by a slight modification of the method and procedure, even when the heart was perfused with a solution of simple salt compositions. These points will be described as supplements to the author's previous method.

\section{Supplements to the Previous Method}

Reduction of the oscillatory pressure-variations due to the artificial perfusion generator ( $A P G)$. The reader is referred to Fig. 1 in the previous report. ${ }^{1}$ In order to minimize artificial pressure-variations, a pressure-modulating reservoir is inserted on the way to the perfusate inflow tube $P i$. The reservoir is a vessel resembling the pulmonary flow-meter $(B)$, which is provided with three tubes: a first tube for collecting the perfusate from the artificial perfusion generator $(A P G)$, a second, the lower end of which reaching the bottom of the reservoir, for flowing the collected perfusate forward, and a third for regulating the quantity of the perfusate and adequately maintaining the air-space in the reservoir. This ar-

Received for publication. February 23, 1967. 
rangement serves to decrease the oscillatory pressure variations sufficiently by minimizing their direct influence upon the manometric recording, and perhaps also upon the heart activities.

Increase of the pulmonary venous flow. For making this increase easy, when necessary, a side channel, in addition to $P i s_{2}$, is provided at the starting portion of the side channel $P i_{s_{1}}$ and connected with the pulmonary venous passage at the site near the heart.

Perfusate. Krebs-Henseleit's solution (without blood) is used, which is saturated with a gas mixture containing $95 \% \mathrm{O}_{2}$ and $5 \% \mathrm{CO}_{2}$.

Gas resemoir. A reservoir of about 10 liters in capacity has two tubes through an air-tight stopper. One is connected with the free end of the respiratory ventile arrangement ( $\mathrm{riv}$ ) through a tube containing some quantity (about $5 \mathrm{ml}$ ) of the perfusate with phenol red and a $\mathrm{Hg}$-manometer, and the other is connected with the air-outflow tube of the heart container $(A)$.

By working the aerating bulb $\left(p_{4}\right)$ of the pulse generator $(P G)$, the gas is introduced into the perfusate reservoir $(C)$. After aerating the perfusate, the gas escapes from the reservoir through the outflow tube $(r x)$. The bubble-remover $r x b$ $r x_{1}$ is omitted. The ontflow gas mixed with bubbled perfusate is introduced directly into the heart container $(A)$ through an inner tube of a stopper at the place of $r h_{2}$. The gas in the container flows out through the outer tube of the stopper, and then goes back into the gas reservoir. The connections of the tube $r x p$ with $r p, r h_{1}$ and $r h_{2}$ are omitted, while the connection between $r$, and $r h_{1}$ is maintained.

The bubbled perfusate which flows into the heart container and is collected in the bottom can easily be sucked out through the pulmonary flow-meter into the perfusate container.

\section{Experimental Procedure}

After the isolated heart is applied to the apparatus, four parallel recordings of pressure in the pulmonary artery, aorta, vena cava and pulmonary vein are taken at every 15 minutes. At each recording, the pressure tracings are taken: 1) during artificial perfusion with working pulse generator, 2) during artificial perfusion, the pulse generator being omitted, and 3) during continued artificial perfusion, the perfusate inflow into the left and right atria being made to increase and the pulmonary outflow being made to decrease by increasing the resistance.

During the whole experimental period, the pulse generator is kept working except at the time of recording.

Sometimes, the total flow through the right heart and the coronary flow are measured by means of the pulmonary flow-meter.

\section{Example}

$11 \mathrm{~h} 40 \mathrm{~min}$ : Application of the heart to the apparatus.

11 h $55 \mathrm{~min}$ : First kymographic tracing (artificial inflow pressure $80-90 \mathrm{mmHg}$ ); total flow 
$10 \mathrm{ml}$ in $5 \mathrm{sec}$, pulmonary flow $5 \mathrm{ml}$ in $13 \mathrm{sec}$ (Fig. 1, I).

$12 \mathrm{~h} 55 \mathrm{~min}$ : 5th tracing (artificial inflow pressure $80-90 \mathrm{~mm} \mathrm{Hg}$ ); total flow $10 \mathrm{ml}$ in 5 sec, pulmonary flow $5 \mathrm{ml}$ in $6 \mathrm{sec}$ (Fig. 1, II).

$13 \mathrm{~h} 55 \mathrm{~min}$ : Total flow $10 \mathrm{ml}$ in 5 sec, pulmonary flow $4 \mathrm{ml}$ in 8 sec.

$14 \mathrm{~h} 25 \mathrm{~min}$ : 11 th tracing (artificial inflow pressure $100 \mathrm{~mm} \mathrm{Hg}$ ) (Fig. 1, III).

I

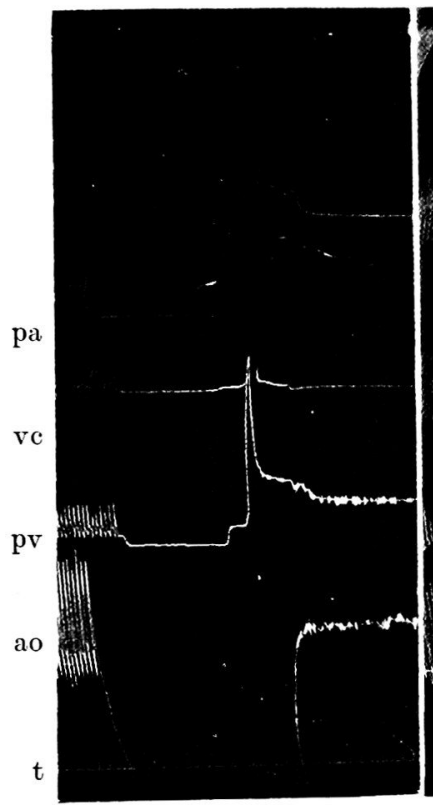

$\uparrow \uparrow \uparrow$

a b c
II

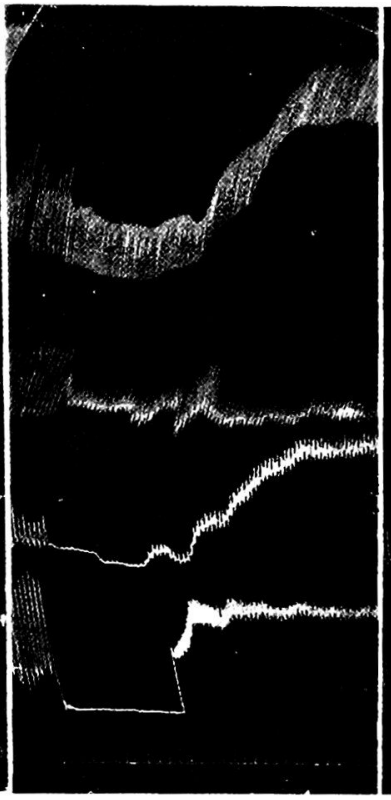

$\uparrow \uparrow \uparrow$
III

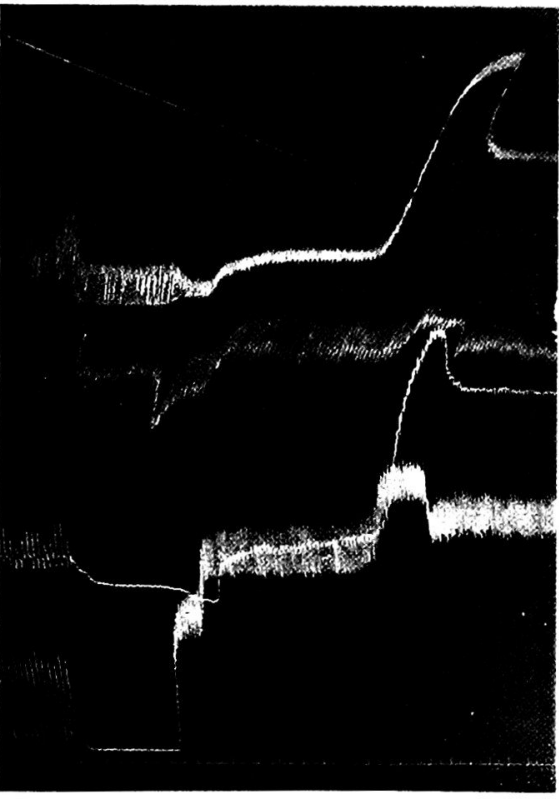

$\uparrow \uparrow \uparrow \uparrow$

a $\quad \mathrm{b} \quad \mathrm{c}$

Fig. 1. Simultaneous tracings of pressure in pulmonary artery (pa), vena cava (vc), pulmonary vein (pv) and aorta (ao) of an isolated rabbit-heart. I: At $11 \mathrm{~h} 55 \mathrm{~min}$. II: At $12 \mathrm{~h} 55 \mathrm{~min}$. III: At $14 \mathrm{~h} 25 \mathrm{~min}$. Time (t) in $3 \mathrm{sec}$.

a-part: During application of $A P G$ and $P G$.

b-part: During application of $A P G$ ( $P G$ omitted).

c-part: During application of $A P G$, venous inflow into the vena cava and pulmonary vein was made to increase, and pulmonary outflow from the right ventricle was made to decrease by increasing the resistance.

Of the tracings, the first, the middle and the last ones are shown in Fig. 1. Heart activities manifested in the first tracing (Fig. 1, I) taken 15 minutes after the application to the perfusion apparatus are weak compared with those at later periods, perhaps on account of insufficient pulmonary flow. The activities one hour after the heart application (5th tracing; Fig. 1, II) do not seem to be much different from those after 3 hours (11th tracing; Fig. 1, III).

\section{Comment}

The author has always observed that the isolated heart applied to the perfusion apparatus does not pulsate, if the left atrium is dilated too much by increased 
perfusate inflow, especially when it is exposed out of the pericardium. Therefore, much care is taken in every perfusion experiment, not to expose the atria and not to cause too much inflow into them. The experiments shown in this paper indicate in general that such activities of both atria as observed in the previous report (lower two tracings in Fig. 1) become hardly perceivable, when the pulse generator ceases to work, and the atria begin to pulsate actively when they are filled with perfusate to an appropriate extent. Under this condition all the tracings (the pulmonary artery, aorta, vena cava and pulmonary vein) exhibit almost parallel changes. However, the heart diminishes its activities immediately after the artificial perfusion procedures are discontinued. In spite of this the heart keeps the potentiality to recover its activity and can manifest it when the artificial perfusion is started again.

Systematic investigations on the relation between the arterial and venous sides of the heart can be made with the present method.

In the present method, a gas reservoir containing $95 \% \mathrm{O}_{2}$ and $5 \% \mathrm{CO}_{2}$ (about 10 liters) is used for the whole experimental period of three hours. The reddish tint of phenol red in the test tube which has been inserted at the end of the outflow gas tube immediately before the gas reservoir, tends to fade along with the increase of $\mathrm{CO}_{2}$ release. Changes in the gas components due to respiration of the heart at any experimental period can be measured with any suitable method.

\section{Acknowledgment}

The author wishes to express his gratitude to Prof. I. Tanaka, Director of the Department and to Dr. M. Yamada, Director of the Kumamoto National Hospital, for giving the author many facilities in carrying out the present work. Thanks are also due to Mr. M. Sato for his technical help in the experimental procedures, and to Dr. Y. Simada for his preparation of perfusion tluid and gas mixture.

\section{Reference}

1) Kodama, $\mathrm{S}$. A method of perfusing an isolated rabbit-heart, with simultaneous pressure-recordings of the aorta, vena cava, pulmonary artery and pulmonary vein. Tohoku J. exp. Med., 1966, 90, 97-103.

\section{Erratum}

In vol. 90, p. 99. line 17, for Aerating system ${ }^{6}$ read Aerating system, and p. 101, Fig. 1, 2 and $\mathrm{I} 2$ lines from bottom, for $r x^{\prime}$ read $r x_{1}$. 